\title{
Limits of the Classical Concept of Concentration
}

\author{
Marco Maioli $^{\mathrm{a}}$, Gyula Varadi ${ }^{\mathrm{b}}$, Róbert Kurdi ${ }^{\mathrm{c}}$, Luciano Caglioti ${ }^{\mathrm{d}}$, Gyula Pályi ${ }^{\mathrm{e}, *}$ \\ ${ }^{a}$ Department of Mathematics, University of Modena and Reggio Emilia, Via Campi 213/B, I-
}

41125 Modena, Italy; marco.maioli@unimore.it

${ }^{b}$ Inpellis, Inc., 100 Cummings Center, Suite 243C, Beverly, MA 01915-6133, USA;

gvaradi@inpellis.com

${ }^{c}$ Institute of Environmental Engineering, University of Pannonia, Egyetem u. 10, H-8200

Veszprém, Hungary; kurdir@uni-pannon.hu

${ }^{d}$ Department of Chemistry and Technology of Biologically Active Compounds, University "La

Sapienza"-Roma, P.le A. Moro 5, I-00185 Roma, Italy; luciano.caglioti@uniroma1.it

${ }^{e}$ Department of Life Sciences, University of Modena and Reggio Emilia, Via Campi 103, I-

41125 Modena, Italy; gyula.palyi@unimore.it

\section{Additional Supporting Information (ASI-1)}

A binomial random variable $X(n ; p)$, with size $n$ and probability $p$, has mean $n \cdot p$ and variance $n p(1-p)$. It represents the number of successes in $n$ independent trials. By varying (or scaling) in suitable ways its parameters $n$ and $p$, three asymptotic results concern $X_{n}$, namely: the law of large numbers, the normal or Gaussian approximation, the Poisson approximation.

By the law of large numbers, the relative frequency $\frac{X(n, p)}{n}$ converges to the probability $p$ itself as $n \rightarrow \infty$. It means that the relative frequency of successes is quite likely to be very close to $p$.

By the "central limit theorem", the fraction $\frac{X(n, p)-n \cdot p}{\sqrt{n p(1-p)}}$ converges in distribution to a normal standard variable (i.e. to a Gaussian variable with mean zero and variance 1) as $n \rightarrow \infty$.

While the preceding asymptotic results hold for fixed parameter $p$, the Poisson approximation holds with sequences of $p$ 's small relative to $n$ : as $n \rightarrow \infty$ and $p_{n} \rightarrow 0$ with the product $n \cdot p \rightarrow \mu$ for some $\mu>0, X\left(n, p_{n}\right)$ converges to a Poisson random variable with mean $\mu$ :

$$
P\left[X\left(n, p_{n}\right)=k\right] \rightarrow P[Y=k]=e^{-\mu} \frac{\mu^{k}}{k !}
$$

for any $\mathrm{k}=0,1,2, \ldots$.

Thus the occupancy number of molecules in a given small sample, which is always binomial, can be more or less near a Poissonian law, depending on the relative variation of $n$, 
p. When it becomes almost Gaussian, then the concentration can be considered as a continuous variable.

For more detailed discussion see:

Ash, R. Real Analysis and Probability. Academic Press, San Diego (CA), 1991. pp.: 234-348.

\section{Additional Supporting Information (ASI-2)}

Code for the computation of mean standard deviation and relative error of the e.e. in a sample of volume V.p with $\mathrm{p}=0.001$ and particle number cca. $<800000$.

error $<$-function(n,p) \{

$\mathrm{m}<-\mathrm{n} / 2$

$\operatorname{Prob}<-\operatorname{matrix}\left(\operatorname{rep}\left(0,(\mathrm{~m}+1)^{\wedge} 2\right), \mathrm{m}+1, \mathrm{~m}+1\right)$

$\mathrm{ZZ}<-$ matrix $\left(\operatorname{rep}\left(0,(\mathrm{~m}+1)^{\wedge} 2\right), \mathrm{m}+1, \mathrm{~m}+1\right)$

$\mathrm{ZZ2<-matrix}\left(\operatorname{rep}\left(0,(\mathrm{~m}+1)^{\wedge} 2\right), \mathrm{m}+1, \mathrm{~m}+1\right)$

\# values of number of particles $\mathrm{X}, \mathrm{Y}$ go from 0 to $\mathrm{m}$,

\# while indices from 1 to $\mathrm{m}+1$

for (i in $1:(m+1))$

for $(\mathrm{k}$ in $1:(\mathrm{m}+1))$

$\operatorname{Prob}[\mathrm{i}, \mathrm{k}]<-$ dbinom $(\mathrm{i}-1, \mathrm{~m}, \mathrm{p})^{*}$ dbinom $(\mathrm{k}-1, \mathrm{~m}, \mathrm{p})$

\#i.e.: $\operatorname{Prob}[\mathrm{i}, \mathrm{k}]<-\operatorname{choose}(\mathrm{m}, \mathrm{i}-1, \mathrm{p})^{*} \operatorname{choose}(\mathrm{m}, \mathrm{k}-1)^{*} \mathrm{p}^{\wedge}(\mathrm{i}+\mathrm{k}-2)^{*}(1-\mathrm{p})^{\wedge}(\mathrm{n}+2-\mathrm{i}-\mathrm{k})$

$\mathrm{Z} 00<-0$

$\mathrm{Z} 01<-\operatorname{abs}(0-1) /(0+1)$

$\mathrm{Z} 10<-\operatorname{abs}(1-0) /(1+0)$

\# define $\mathrm{ZZ}$ as a translated of $\mathrm{Z}=|\mathrm{X}-\mathrm{Y}| /(\mathrm{X}+\mathrm{Y})$

\# indeed indices must go from 1 to $\mathrm{m}+1$

$\mathrm{ZZ}[1,1]<-\mathrm{Z} 00$

$Z Z[1,2]<-Z 01$ 
$\mathrm{ZZ}[2,1]<-\mathrm{Z} 10$

for ( $\mathrm{i}$ in $2:(\mathrm{m}+1))$

for $(k$ in $2:(m+1))$

$\mathrm{ZZ}[\mathrm{i}, \mathrm{k}]<-\operatorname{abs}(\mathrm{i}-\mathrm{k}) /(\mathrm{i}+\mathrm{k}-2)$

\#define the ZZ squared

$\mathrm{ZZ2}<-\mathrm{ZZ}^{\wedge} 2$

\# compute mean of $\mathrm{Z}$ and mean of $\mathrm{Z}$ squared

$\mathrm{EZ}<-\operatorname{sum}(Z Z *$ Prob)

EZ2<-sum(ZZ2*Prob)

\# compute the standard deviation of $\mathrm{Z}$ and the relative error

$\operatorname{sdZ}<-\operatorname{sqrt}\left(\mathrm{EZ2}-(\mathrm{EZ})^{\wedge} 2\right)$

$\mathrm{reZ}<-\mathrm{sdZ} / \mathrm{EZ}$

$\mathrm{c}(\mathrm{EZ}, \mathrm{sdZ}, \mathrm{reZ})$

Code for the computation of mean standard deviation and relative error of the e.e. in a sample of volume V.p with $\mathrm{p}=0.001$ and particle number cca. $>800000$.

error_approx $<-$ function(n,p) \{

$\mathrm{m}<-\mathrm{n} / 2$

$\mathrm{r} 2<-\operatorname{round}(\mathrm{m} * \mathrm{p}+5 * \operatorname{sqrt}(\mathrm{m} * \mathrm{p} *(1-\mathrm{p})))$

$\mathrm{r} 1<-$ round $\left(\mathrm{m}^{*} \mathrm{p}-5 * \operatorname{sqrt}\left(\mathrm{m}^{*} \mathrm{p} *(1-\mathrm{p})\right)\right)$

$\mathrm{r}<-\mathrm{r} 2-\mathrm{r} 1$

$\operatorname{Prob}<-\operatorname{matrix}\left(\operatorname{rep}\left(0,(\mathrm{r}+1)^{\wedge} 2\right), \mathrm{r}+1, \mathrm{r}+1\right)$

$\mathrm{ZZ}<-$ matrix $\left(\operatorname{rep}\left(0,(\mathrm{r}+1)^{\wedge} 2\right), \mathrm{r}+1, \mathrm{r}+1\right)$

$\mathrm{ZZ2}<-\operatorname{matrix}\left(\operatorname{rep}\left(0,(\mathrm{r}+1)^{\wedge} 2\right), \mathrm{r}+1, \mathrm{r}+1\right)$

\# values of number of particles $\mathrm{X}, \mathrm{Y}$ go from 0 to $\mathrm{r}$, 
\# while indices in this software go from 1 to $\mathrm{r}+1$

for (i in $1:(r+1))$

for $(\mathrm{k}$ in $1:(\mathrm{r}+1))$

$\operatorname{Prob}[\mathrm{i}, \mathrm{k}]<-$ dbinom $(\mathrm{r} 1+\mathrm{i}-1, \mathrm{~m}, \mathrm{p}) * \operatorname{dbinom}(\mathrm{r} 1+\mathrm{k}-1, \mathrm{~m}, \mathrm{p})$

\#that is: $\operatorname{Prob}[\mathrm{i}, \mathrm{k}]<-$ choose $(\mathrm{m}, \mathrm{r} 1+\mathrm{i}-1, \mathrm{p})^{*} \operatorname{choose}(\mathrm{m}, \mathrm{r} 1+\mathrm{k}-1)^{*} \mathrm{p}^{\wedge}(\mathrm{r} 1+\mathrm{i}+\mathrm{r} 1+\mathrm{k}-2)^{*}(1-\mathrm{p})^{\wedge}(\mathrm{m}+2-\mathrm{i}-$

r1-k-r1)

\# define $\mathrm{ZZ}$ as a translated of $\mathrm{Z}=|\mathrm{X}-\mathrm{Y}| /(\mathrm{X}+\mathrm{Y})$

\# indices must go from 1 to $\mathrm{r}+1$

for (i in $1:(r+1))$

for $(\mathrm{k}$ in $1:(\mathrm{r}+1))$

$\mathrm{ZZ}[\mathrm{i}, \mathrm{k}]<-\operatorname{abs}(\mathrm{i}-\mathrm{k}) /(\mathrm{r} 1+\mathrm{i}+\mathrm{r} 1+\mathrm{k}-2)$

\#define the ZZ squared

$\mathrm{ZZ2}<-\mathrm{ZZ}^{\wedge} 2$

\# compute mean of $\mathrm{Z}$ and mean of $\mathrm{Z}$ squared

EZ<- sum(ZZ*Prob)

EZ2<-sum(ZZ2*Prob)

\# compute the standard deviation of $\mathrm{Z}$ and the relative error

$\operatorname{sdZ}<-\operatorname{sqrt}\left(\mathrm{EZ2}-(\mathrm{EZ})^{\wedge} 2\right)$

$\mathrm{reZ}<-\mathrm{sdZ} / \mathrm{EZ}$

$\mathrm{c}(\mathrm{EZ}, \mathrm{sdZ}, \mathrm{reZ})$

Code for the computation of mean standard deviation and relative error of the e.e. in a sample of volume V.p with $\mathrm{p}=0.001$ for odd particle numbers with one particle excess from one of the enantiomers. 
error_uneven $<$-function(n,p) \{

m<-round(n/2)

$\mathrm{r} 2<-\operatorname{round}\left(\mathrm{m}^{*} \mathrm{p}+5 * \operatorname{sqrt}\left(\mathrm{m}^{*} \mathrm{p} *(1-\mathrm{p})\right)\right)$

$\mathrm{r} 1<-$ round $\left((\mathrm{m}+1) * \mathrm{p}-5 * \operatorname{sqrt}\left((\mathrm{m}+1)^{*} \mathrm{p} *(1-\mathrm{p})\right)\right)$

$r<-r 2-r 1$

Prob<-matrix $(\operatorname{rep}(0,(\mathrm{r}+1) *(\mathrm{r}+2)), \mathrm{r}+1, \mathrm{r}+2)$

$\mathrm{ZZ}<-$ matrix $(\operatorname{rep}(0,(\mathrm{r}+1) *(\mathrm{r}+2)), \mathrm{r}+1, \mathrm{r}+2)$

$\mathrm{ZZ2}<-$ matrix $(\operatorname{rep}(0,(\mathrm{r}+1) *(\mathrm{r}+2)), \mathrm{r}+1, \mathrm{r}+2)$

\# values of number of particles X,Y go from 0 to $\mathrm{r}$,

\# while indices in this software go from 1 to $\mathrm{r}+1$

for (i in $1:(r+1))$

for $(\mathrm{k}$ in $1:(\mathrm{r}+2))$

$\operatorname{Prob}[\mathrm{i}, \mathrm{k}]<-$ dbinom $(\mathrm{r} 1+\mathrm{i}-1, \mathrm{~m}, \mathrm{p}) * \operatorname{dbinom}(\mathrm{r} 1+\mathrm{k}-1, \mathrm{~m}+1, \mathrm{p})$

\#that is: $\operatorname{Prob}[\mathrm{i}, \mathrm{k}]<-$ choose $(\mathrm{m}, \mathrm{r} 1+\mathrm{i}-1, \mathrm{p})^{*} \operatorname{choose}(\mathrm{m}, \mathrm{r} 1+\mathrm{k}-1)^{*} \mathrm{p}^{\wedge}(\mathrm{r} 1+\mathrm{i}+\mathrm{r} 1+\mathrm{k}-2)^{*}(1-\mathrm{p})^{\wedge}(\mathrm{m}+2-\mathrm{i}-$

r1-k-r1)

\# define $\mathrm{ZZ}$ as a translated of $\mathrm{Z}=|\mathrm{X}-\mathrm{Y}| /(\mathrm{X}+\mathrm{Y})$

\# indices must go from 1 to $\mathrm{r}+1$

for (i in $1:(r+1))$

for $(\mathrm{k}$ in $1:(\mathrm{r}+2))$

$\mathrm{ZZ}[\mathrm{i}, \mathrm{k}]<-\operatorname{abs}(\mathrm{i}-\mathrm{k}) /(\mathrm{r} 1+\mathrm{i}+\mathrm{r} 1+\mathrm{k}-2)$

\#define the ZZ squared

$\mathrm{ZZ2}<-\mathrm{ZZ}^{\wedge} 2$

\# compute mean of $\mathrm{Z}$ and mean of $\mathrm{Z}$ squared 
$\mathrm{EZ}<-\operatorname{sum}(Z Z *$ Prob)

EZ2<-sum(ZZ2*Prob)

\# compute the standard deviation of $\mathrm{Z}$ and the relative error

$\operatorname{sdZ}<-\operatorname{sqrt}\left(\mathrm{EZ2}-(\mathrm{EZ})^{\wedge} 2\right)$

$\mathrm{reZ}<-\mathrm{sdZ} / \mathrm{EZ}$

$\mathrm{c}(\mathrm{EZ}, \mathrm{sdZ}, \mathrm{reZ})$

\section{Additional Supporting Information (ASI-3)}

Additional relevant references

Single chiral molecules, origins of life and of biological chirality

Caglioti, L.; Hajdu, C.; Holczknecht, O.; Zékány, L.; Zucchi, C.; Micskei, K.; Pályi, G. Racemates and the Soai Reaction. Viva Orig. 2006, 34, 62-80.

Fuss, W. Biological Homochirality as a Result of a Single Event. Colloids Surf. B Biointerface 2009, 74, 498-503.

Garay, J. Active Centrum Hypothesis. The Origin of Chiral Homogenity and the RNA-World. BioSystems 2011, 103, 1-12.

Caglioti, L.; Pályi, G. Single Chiral Molecule as Possible Starting Element of Complex Chiral Systems. Rend Lincei, Sci. Fis. Nat. 2013, 24, 191-196.

Kafri, R.; Markovitch, O.; Lancet, D. Spontaneous Chiral Symmetry Breaking in Early Molecular Networks. Biol. Direct 2010, 5, Article No. 38.

Pavlov, V. A.; Klabunovski, E. I.; Homochirality. Origin in Nature: Possible Versions. Curr. Org. Chem. 2014, 18, 93-114.

Morneau, B. N.; Kubala, J. M.; Barratt, C.; Schwatz, P.M. Analysis of a Chemical Model System Leading to Chiral Symmetry Breaking: Implications for the Evoilution of Homochirality. J. Math. Chem. 2014, 52, 268-282. 


\section{Analysis at very low concentrations or very small volumes}

Han, X. X.; Huang, G. G.; Zhao, B.; Ozaki, Y. Label-Free, Highly Selective Detection of Proteins in Aqueous Solutions Using Surface-Enhanced Raman Scattering. Anal. Chem. 2009, 81, 3329-3333.

Jiang, D.; Zhang, Q.; Shen, X.; Wang, L.; Jiang, W. Solid-Phase Single Molecule Counting of Antibody Binding to Supported Protein Layers Surface with Low Non-Specific Adsorption. Talanta 2010, 82, 1003-1009.

Liu, J.; Liu, Y.; Yang, X.; Wang, K.; Wang, Q.; Shi, H.; Li, L. Energy Transfer-Based Fluorescent Sensing through Aptameter-Programed Self Assembly of Quantum Dots. Anal. Chem.2013, 85, 11121-11128.

Rivnak, A. J.; Rissin, D. M.; Kan, C. W.; Seng, L.; Fishburn, M. W.; Piech, T.; Campbell, T. G.; Du Pont, D. R.; Gardel, M.; Sullivan, S.; Pink, B. A.; Cabrera, C. G.; Farnier, D.R.;

Duffy, D. C. A Fully-Automated, Six-plex Single Molecule Immunoassay for Measuring Citokines in Blood. J. Immunol. Methods 2015, 424, 20-27.

\section{Micro needles}

Prausnitz, M. R. Microneedles for Transdermal Drug Delivery. Adv. Drug. Deliv. Rev. 2004, $56,581-587$.

Gill, H. S.; Prausnitz, M. R. Coated Microneedles for Transdermal Delivery. J. Control Release 2007, 117, 227-237.

Chen, X.; Kis, A.; Zettl, A.; Bertozzi, C. R. A Cell Nano-Injector Based on Carbon Nanotubes. Proc. Natl. Acad. Sci. USA 2007, 104, 8218-8222.

Abate, A. R.; Hung, T.; Mary, P.; Agresti, J. J.; Weitz, D. A. High-Throughput Injection with Microfluidics Using Picoinjectors. Proc. Natl. Acad. Sci. USA 2010, 107, 19163-19166. 
Seger, R. A.; Actis, P.; Penfold, C.; Maalouf, M.; Vilozny, B.; Pourmand, N. Voltage Controlled Nano-Injection System for Single Cell Surgery. Nanoscale 2012, 4, 5843-5846.

Mahboobi, S. H.; Taheri, A.; Pishkenari, H. N.; Meghdari, A.; Hemmat, M. Cellular Injection Using Carbon Nanotube: A Molecular Dynamics Study. Nano 2015, 10, No. 1550025.

\section{Nanopipettes}

Deng, X. L.; Takami, T.; Son, J. W.; Kawai, T.; Park, B. H. Ion Current Oscillation in Glass Nanopipettes. J. Phys. Chem. C 2012, 116, 14857-14862.

Kang, E. J.; Takami, T.; Deng, X. L.; Son, J. W.; Kawai, T.; Park, B. H. Improved IonSelective Detection Method Using Nanopipette with Poly(vinyl chloride)-Based Membrane. J. Phys. Chem. B. 2014, 118, 5130-5134.

Yu, Y.; Noel, J.-M.; Mirkin, M. V.; Gao, Y.; Mashtalir, O.; Friedman, G.; Gogotsy, Y. Carbon Pipette-Based Electrochemical Nanosampler. Anal. Chem. 2014, 86, 3365-3372.

Shi, W.; Sa, N.; Thakar, R.; Baker, L. A. Nanopipette Delivery: Influence of Surface Change. Analyst 2015, 140, 4835-4842.

Larson, J. M.; Bharath, S. C.; Cullen, W. G.; Reutt-Robey, J. E. Scanning MWCNTNanopipette and Probe Microscopy: Li Patterning and Transport Studies. Small 2015, 11, 4946-4958.

\section{Importance and history of asymmetric catalysis}

Noyori, R. Asymmetric Catalysis in Organic Synthesis. Wiley, New York, 1994.

Berhessel, A.; Gröger, H. Asymmetric Organocatalysis. Wiley-VCH, Weinheim, 2005.

Organometallic Chirality; Pályi, G.; Zucchi, C.; Caglioti, L. Eds.; Mucchi-Accad. Nazl. Sci. Lett. Arti, Modena, 2008.

Debré, P. Louis Pasteur. Flammarion, Paris, 1994.

Dubos, R. Pasteur and Modern Science, ASM Press, Washington DC, USA, 1998. 
Mislow, K. Molecular Chirality. Top. Stereochem. 1999, 22, 1-82.

Asymmetric autocatalysis induced by very low concentrations of chiral additives

Shibata, T.; Yonekubo, S.; Soai, K. Practically Perfect Asymmetric Autocatalysis with (2Alkynyl-5-pyrimidyl)alkanols. Angew. Chem., Int. Ed. 1999, 38, 659-661.

Micskei, K.; Maioli, M.; Zucchi, C.; Caglioti, L.; Pályi, G. Generalization Possibilities of Autocatalytic Absolute Enantioselective Synthesis. Tetrahedron: Asymmetry 2006, 17, 29602962.

Sato, I.; Osanai, S.; Kadowaki, K.; Sugiyama, T.; Shibata, T.; Soai, K. Asymmetric Autocatalysis of Pyrimidyl Alkanol Induced by Optically Active 1,1'-Binaphthyl an Atropisomeric Hydrocarbon Generated by Spontaneous Resolution on Crystallization. Chem. Lett. 2002, 2, 168-169.

Sato, I.; Ohno, A.; Aoyama, Y.; Kasahara, T.; Soai, K. Asymmetric Autocatalysis Induced by Chiral Hydrocarbon [2,2]-Paracyclophanes. Org. Biomol. Chem. 2003, 1, 244-246.

Kawasaki, T.; Hatase, K.; Fujii, Y.; Jo, K.; Soai, K.; Pizzarello, S. The Distribution of Chiral Asymmetry in Meteorites: An Investigation Using Asymmetric Autocatalytic Chiral Sensors. Geochim. Cosmochim. Acta 2006, 70, 5395-5402.

Kawasaki, T.; Suzuki, K.,; Licandro, E.; Bossi, A.; Maiorana, S.; Soai, K. Enantioselective Synthesis Induced by Tetrathia-[7]-helicenes in Conjunction with Asymmetric Autocatalysis. Tetrahedron: Asymmetry 2006, 17, 2050-2053.

Shibata, T.; Iwahashi, K.; Kawasaki, T.; Soai, K. Chiral Secondary Alcohol-Induced Asymmetric Autocatalysis: Correlation between the Absolute Configuration of the Chiral Initiators and the Product. Tetrahedron: Asymmetry 2007, 18, 1759-1762.

Kawasaki, T.; Shimizu, M.; Nishiyama, D.; Ito, M.; Ozawa, H.; Soai, K. Asymmetric Autocatalysis Induced by Meteoritic Amino Acids with Hydrogen Isotope Chirality. Chem. Commun. 2009, 4396-4398. 
Matsumoto, A.; Oji, S.; Takano, S.; Tada, K.; Kawasaki, T.; Soai, K. Asymmetric Autocatalysis Triggered by Oxygen Isotopically Chiral Glycerin. Org. Biomol. Chem. 2013, 11, 2928-2931.

Ozaki, H.; Tada, K.; Harada, S.; Ayugase, T.; Ozawa, H.; Kawasaki, T.; Matsumoto, A.; Soai,

K. Asymmetric Autocatalysis Triggered by Nitrogen Isotope Chirality. $3^{\text {rd }}$ International Symposium on the Soai Reaction and Related Topic. Felsőmocsolád, Hungary, September 2-5, 2015. Abstracts pp.: 24-25.

\section{Stochastic description of asymmetric autocatalysis}

Lente, G. Stochastic Kinetic Models of Chiral Autocatalysis: A General Tool for the Quantitative Interpretation of Total Asymmetric Synthesis. J. Phys. Chem. A 2005, 109, 11058-11063.

Lente, G. Stochastic Interpretation of the Asymmetry of Enantiomeric Distribution Observed in the Absolute Asymmetric Soai Reaction. Tetrahedron: Asymmetry 2011, 22, 1595-1599.

Lente, G. Stochastic Modeling of the Soai Reaction. In: The Soai Reaction and Related Topic (Pályi, G.; Zucchi, C.; Caglioti, L., Eds.). Artestampa-Accad. Naz. Sci. Lett. Arti, Modena, 2012. pp.: 123-147.

Érdi, P.; Lente, G. Stochastic Chemical Kinetics. Springer, Heidelberg, 2014. 